\title{
Recurrent Meningitis in an Adult Due to Nontypable Haemophilus influenzae
}

Case history. The patient, who had been born in 1940, had smoked one pack of cigarettes daily since adolescence. In 1972 she was involved in a car accident, a few days after which she presented to an emergency room complaining of headaches. Roentgenograms were normal. Approximately six months later she started to suffer from a runny nose. Attempts by several physicians to cure this presumed "rhinitis" were unsuccessful. She was admitted under our care in April 1982 after a 24-hr period of fever and headache. Lumbar puncture revealed cloudy CSF with 2,600 leukocytes $/ \mathrm{mm}^{3}$ and $90 \%$ PMNs; cultures yielded Haemophilus influenzae. Treatment was started with penicillin and changed to ampicillin after culture results became known, and the patient made an uneventful recovery.

The patient declined subsequent neurosurgical consultation and remained well until March 1983, when she developed symptoms of an upper-respiratory-tract infection, and fever and violent headaches led to hospitalization. Again, CSF was purulent, and cultures revealed $H$ influenzae. Recovery with ampicillin was excellent.

Two days after the second episode of meningitis, unilateral rhinorrhea became profuse, exceeding $1 \mathrm{ml} / \mathrm{min}$, the fluid having the chemical composition of CSF. Seven days later, rhinorrhea ceased. The patient underwent neurosurgical exploration 17 days after the second admission, but the neurosurgeons were unable to visualize a fistula. Serum immunoglobulin and complement levels were normal.

Both microorganisms isolated from CSF were identified as $H$ influenzae by standard methods [1]. Neither produced iridescent colonies on fresh Levinthal agar or formed precipitin bands by CIE with monospecific antisera to $H$ influenzae types a-f (Burroughs Wellcome, Cornwallis, NC). The outer-membrane proteins of the isolates were subjected to SDS-PAGE. The electrophoretic patterns were clearly different, indicating that the organisms were of different strains; however, both were of the same Kilian biotype II.

Unique features. We were unable to find another

This work was supported in part by Public Health Service grant AI 17572 from the National Institute of Allergy and Infectious Diseases of the National Institutes of Health.

Please address requests for reprints to Dr Bernard J. Hirschel, Division des Maladies Infectieuses, Départment de Médecine, Hôpital Cantonal Universitaire, 1211-Geneva ' 4, Switzerland. case in the literature of an adult with two episodes of Haemophilus-related meningitis caused by encapsulated or nonencapsulated (nontypable) organisms; however, a similar case has been noted in a 15 -year-old boy with a ventriculo-peritoneal shunt and suspected cribiform plate abnormality who had two episodes of meningitis in a 10-week period [1]. Evidence for the existence of an intermittent fistula was strong, despite the negative results of the neurosurgical exploration. Since nasopharyngeal carriage of $H$ influenzae by humans is frequent, especially in smokers, and the strains are diverse, different nonencapsulated strains may well have migrated through the suspected fistula of our patient on separate occasions and entered the CNS from the nasopharynx.

Conclusions. In the context of a clinically diagnosed cerebrospinal-nasal fistula, humoral immunity is probably ineffective in preventing recurrent episodes of meningitis, even if the antibody response to the first infection is capable of conferring protection against bacteremia caused by the heterologous strain associated with the second episode. However, recent evidence indicates that protection resulting from anti-outer membrane-protein antibody may be strain specific for both type b [2] and for nontypable organisms. Therefore, lack of a protective antibody response against the heterologous strain causing the second infection may also have been a factor in the recurrence.

Bernard J. Hirschel, Raymond Auckenthaler, Stephen J. Barenkamp, Dan M. Granoff Division des Maladies Infectieuses, Départment de Médecine, Hôpital Cantonal Universitaire, Geneva, Switzerland; and Edward Mallinckrodt Department of Pediatrics, Washington University School of Medicine, and Division of Infectious Diseases, St Louis Children's Hospital, St Louis, Missouri

\section{References}

1. Barenkamp SJ, Munson RS Jr, Granoff DM. Outer membrane protein and biotype analysis of pathogenic nontypeable Haemophilus influenzae. Infect Immun 1982; 36:535-40

2. Shenep JL, Munson RS Jr, Barenkamp SJ, Granoff DM. Further studies of the role of noncapsular antibody in protection against, experimental Haemophilus influenzae type b bacteremia. Infect Immun 1983;42:257-63 